\title{
Effect of Paclitaxel along with Di allyl sulfide on Nucleic acid and polyamines changes in 7, 12 Di methyl benz(a) anthracene induced skin cancer wistar rats
}

\author{
Dr.N.Muninathan
}

\begin{abstract}
Aim: The purpose of this study is to investigate the changes in the levels of nucleic acid and polyamines and efficacy of combination of paclitaxel along with Di allyl sulfide against skin cancer in experimental animals. Skin cancer is the most common form of human cancer. It is estimated that over 1 million new cases occur annually. The annual rates of all forms of skin cancer are increasing each year, representing a growing public concern. The most common warning sign of skin cancer is a change in the appearance of the skin, such as a new growth or a sore that will not heal. Skin cancer is caused by chemical carcinogens and Papilloma virus infection. Design/Methods: Skin cancer was induced in rats by 7, 12 Di methyl benz(a) anthracene (DMBA) at the dosage of $5 \mu \mathrm{g}$ dissolved in $100 \mu \mathrm{l}$ acetone and administered into experimental animals for 28 weeks. In this study, we demonstrated that combination of paclitaxel and Di allyl sulfide revert the changes in the rats from lethal dose of DMBA within 30 days. Results: The levels of Nucleic acid and polyamines in skin and liver were found to be increased in the cancer bearing animals when compared with control animals. Treatment of Paclitaxel along with Di allyl sulfide to cancer induced animals showed significant decrease in the levels of Nucleic acid when compared with cancer induced animals. Conclusions: The treatment with combination of paclitaxel and Di allyl sulfide effectively reduced nucleic acid and polyamines levels. So, from the obtained results it is concluded that paclitaxel and Di allyl sulfide is capable of restoring the skin architecture.
\end{abstract}

Key words: Paclitaxel, Di allyl sulfide, DMBA, Skin cancer.

\section{Introduction}

Skin cancer is the most common form of human cancer. It is estimated that over 1 million new cases occur annually (American Cancer Society). In India, skin cancers constitute about 1-2\% of all diagnosed cancers. Basal cell carcinoma is the commonest form of skin cancer worldwide, but various studies from India have consistently reported Squamous cell carcinoma as the most prevalent skin malignancy.

PAH's are an important class of chemical carcinogens that are widespread in the ambient environment due to fossil fuel combustion for energy production, transportation and industry . DMBA, a potent PAH recognized as an initiator of both skin and liver cancer (Masaaki Miyata et al). The covalent binding of DMBA metabolites to DNA has been implicated as a critical step in the initiation phase of cancers.

Paclitaxel (Taxol), a naturally occurring antineoplastic agent has shown great promise in the therapeutic management of certain human solid tumors particularly in metastatic breast cancer and malignancy involves skin, lung and refractory ovaries. It is the original member of the taxane group of anticancer drugs derived from the bark and needles of the pacific yew tree "Taxus brevifolia". Paclitaxel's antitumor activity was discovered in1960's during a large scale 35,000 plants-screening program sponsored by the National Cancer Institute (NCI), USA.

Garlic has been applied since time immemorial as a culinary spice and medicinal herb and is an important constituent of traditional Chinese medicine. The chief constituent of garlic is the sulfur compound allicin, produced by crushing or chewing fresh garlic, which in turn produces other sulfur compounds: ajoene, mono-, di-, and tri-allyl sulfides, and vinyldithiins (Ali et al).

Nucleic acids play an important role during neoplastic transformation. During neoplastic condition the abnormalities in DNA increases with degree of malignancy. Since DMBA has been shown to bind to cellular DNA, considerable evidences indicate that such DMBA-DNA interactions are responsible for initiation of carcinogenic process and hence, the determination of DNA is more meaningful in tumorous condition.

The polyamines histamine, putrescine, spermine and spermidine are ubiquitous low molecular weight aliphatic cations that play multifactorial roles in cell growth and differentiation (Schipper et al., 2000). Rapidly growing cells have higher amounts of polyamines and they have stimulating effect on DNA, RNA and protein synthesis. They are also involved in stabilizing membranes and cytoskeletal structures (Canizares et al., 1999). 


\section{Chemicals:}

\section{Materials And Methods}

7,12 Dimethyl benz (a) anthracene and Di allyl sulfide were purchased from Sigma chemical company, USA. All the other chemicals used were of analytical grade.

\section{Animal care and housing:}

Male Wistar rats, 6-8 weeks of age and weighing 150-200g, were used. The animals were procured from Central Animal House Block, Meenakshi Medical College and Research institute, Kanchipuran, Tamil Nadu, India and maintained in a controlled environmental condition of temperature and humidity on alternatively $12 \mathrm{~h}$ light/dark cycles. All animals were fed standard pellet diet (Gold Mohor rat feed, Ms.Hindustan Lever Ltd., Mumbai) and water ad libitum. This research work on wistar male rats was sanctioned and approved by the Institutional Animal Ethical Committee (REG NO. 765/03/ca/CPCSEA).

\section{Experimental Design}

The animals were divided in to six groups of 6 animals each. Group I animals served as control, Group II, III, IV, V as animals treated with DMBA (5 $\mu \mathrm{g})$ per animal in acetone $(100 \mu \mathrm{L})$, three times a week for 28 weeks to induce skin cancer. After tumor induction Group III animals were treated with Paclitaxel $(33 \mathrm{mg} / \mathrm{kg}$ b.wt) once in a week for 4 weeks. Group IV animals were treated with garlic extract of Di allyl sulfide

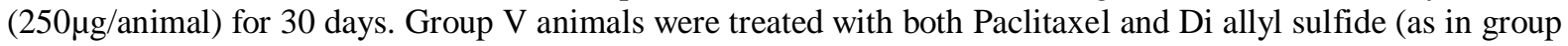
III and group IV). These was Group VI Control animals treated with paclitaxel and Di allyl sulfide for 28 weeks plus 30 days.

After the experimental period of 32 weeks, the animals were sacrificed by cervical decapitation. Extraction of nucleic acids

The nucleic acids were extracted by the method of Schneider (1957).

Known amount of tissues were homogenized in $5.0 \mathrm{ml}$ of ice-cold distilled water using Potter Elvehjem homogenizer with a teflon pestle. $5.0 \mathrm{ml}$ of 5\% TCA was added to the homogenate and this was kept in ice for 30 minutes to allow complete precipitation of proteins and nucleic acids. The mixture was centrifuged and the precipitate obtained was washed thrice with ice cold $10 \%$ TCA. Then it was treated with $95 \%$ ethanol to remove lipids. The final precipitate was heated at $90^{\circ} \mathrm{C}$ for 15 minutes with occasional shaking, which facilitated the quantitative separation of

nucleic acids from protein. The supernatant after centrifugation was used for the estimation of DNA and RNA.

\section{Biochemical analysis}

DNA was estimated by the method of Burton (1956). RNA was estimated by the method of Rawal et al., (1977) and the levels of putrescine and polyamines were assayed by the method of Endo (1978).

\section{Results}

Fig. 1 depicts the effect of paclitaxel along with Di allyl sulfide on the levels of nucleic acids (DNA and RNA) in the skin of control and experimental animals. The levels of both the nucleic acids were found to be significantly ( $\mathrm{p}<0.001)$ increased in the cancer induced group (G-II) when compared with the control group (GI). On treatment with paclitaxel (G-III) and Di allyl sulfide (G-IV) there found to be a significant ( $\mathrm{p}<0.001 ; \mathrm{p}<$ 0.01 ) decrease in the levels of DNA and RNA when compared with cancer bearing animals (G-II). The combination Paclitaxel and Di allyl sulfide $(\mathrm{G}-\mathrm{V})$ treated animals caused a much significant $(\mathrm{p}<0.001)$ decrease in their levels when compared with the cancer-induced group(G-I). There was no significant difference in the nucleic acid levels between the control animals and the control animals treated with the combination of paclitaxel and Di allyl sulfide (G-VI).

The fig. 2 depicts the effect of paclitaxel along with Di allyl sulfide on the levels of nucleic acids (DNA and RNA) in the liver of control and experimental animals. The levels of both the nucleic acids were found to be significantly $(\mathrm{p}<0.001)$ increased in the cancer induced group (G-II) when compared with the control group (GI). Paclitaxel (G-III) and Di allyl sulfide (G-IV) treatment caused a significant ( $p<0.001 ; \mathrm{p}<0.01 ; \mathrm{p}<0.05$ ) decrease in the levels of DNA and RNA when compared with cancer bearing animals (G-II). Cancer bearing animals treated with a combination of paclitaxel and Di allyl sulfide $(\mathrm{G}-\mathrm{V})$ also showed a significant $(\mathrm{p}<0.001)$ decrease in their levels when compared with the cancer-induced group. There was found to be no significant difference in the nucleic acid levels between the control animals and the control animals treated with the combination of paclitaxel and Di allyl sulfide (G-VI).

\section{Polyamines}

The fig. 3 and 4 show the levels of polyamines like histamine, putrescine, spermidine and spermine in the skin and liver of control and experimental animals respectively. The first and second peak corresponds to 
histamine and putrescine. The third and fourth peak corresponds to spermidine and spermine respectively. The levels of polyamines were found to be increased in cancer induced group (G-II) when compared with the control group (G-I). Administration of paclitaxel (G-III) and Di allyl sulfide (G-IV) cause a significant decrease in the levels of polyamines when compared with cancer bearing animals. Cancer bearing group treated with both paclitaxel and Di allyl sulfide (G-V) showed a significant decrease in the activities of the polyamines when compared with the cancer-induced group. There was no alteration in the levels of polyamines in control animals and control animals treated with paclitaxel and Di allyl sulfide (G-VI).

\section{Discussion}

Ellis et al., (1991) have also reported an abnormal amount of DNA and RNA in many cancers including skin carcinoma, which is consistent with present findings. Paclitaxel and DAS decreases the level of DNA and RNA. Combination therapy of paclitaxel and DAS more significantly controlled the nucleic acid synthesis suggesting the enhanced tumoricidal activity of combination chemotherapy.

Polyamines are involved in the development and growth regulation of skin cancer (Leveque et al., 2000). Cells that are stimulated to reproduce demonstrated early increase in biosynthesis and subsequent elevations in polyamine levels. Ornithine decarboxylase, S-adenosyl methionine decarboxylase, spermine synthase and spermidine synthase are the major enzymes in the polyamine biosynthetic pathway. Sunkara et al., (1987) have reported that ODC is directly involved in skin carcinoma cell invasion. It is evident that polyamines are associated intimately to growth processes (Cipolla et al., 1993; stjernborg et al., 1993). The earlier reports show the increased levels of polyamines content in various cancer patients (Mukerjee et al., 1993, Chanda and ganguly, 1988). It was explained that high levels of polyamines are generally associated with the carcinomatous growths as high rate of cell multiplication. The previous experiments with different carcinoma tissues have demonstrated a marked increase in the levels of polyamines and ODC activity confirming their correlation with neoplastic growth and high rate of cell proliferation (Bandyopadhyay and Ganguly, 1999; Farriol et al., 2001).

The cancer animals were associated with a significant increase in tissue nucleic acid content and simultaneous increase in polyamine levels. In the present study, the levels of nuclic acid polyamines were increased in cancer bearing group II animals. Paclitaxel and Di allyl sulfide treatment reduces the levels of polyamine synthesis in cancer bearing animals. This may be due to the inhibition of ODC activity and reduce cell proliferation in skin cancer animals. The reduction of polyamine synthesis induced during chemotherapy is an important event for the effective treatment in skin cancer.

\section{Statistical analysis}

For statistical analysis, one way analysis of analysis of Variance (ANOVA) was used, followed by the Newman-Keuls Multiple Comparison test.

\section{Conclusion}

From the present study, the effect of Paclitaxel- DAS combination proved to be effective chemotherapeutic agent against DMBA induced skin cancer in wistar rats compared to that of paclitaxel or Di allyl sulfide confirmed analyzing the Nucleic acid and polyamines levels in skin and liver tissue.

\section{Reference}

[1] Ali M, Thomson M, Afzal M. (2000). Garlic and onions: their effect on eicosanoid metabolism and its clinical relevance. Prostaglandins Leukot Essent Fatty Acids, 62, 55-73.

[2] Burton K. (1956). A study of the conditions and mechanism of the diphenylamine reaction for the colorimetric estimation of deoxyribonucleic acid. Biochem. J., $62: 315-323$.

[3] Canizares F, Salinas J, Heras M. and Penafiel R. (1999). Prognostic value of ornithine decarboxylase and polyamines in human breast cancer : correlation with clinicopathological parameters. Clinical Research. 5: 2035 - 41.

[4] Chanda R and Ganguly AK (1987). Diamine Oxidase activity and tissue histamine content of human skin, breast and rectal carcinoma. Cancer let, 34; 207-212.

[5] Cipolla B, Guille F, Moulinoux JP, Quemener V, Staerman F, Corbel L and Lobel B. (1993).

[6] Polyamine and prostatic carcinoma : Clinical and therapeutic implications. Eur. Urol., 24: 124 -131.

[7] Ellis CN, Burnette JJ and Sedlack R (1991). Prognostic applications of DNA analysis in solid malignant lesions in humans. Surgery, 173: $329-342$

[8] Endo Y. (1978). A simple and sensitive method of analysis of histamine, putrescine and polyamines without the use of an amino acid analyser. Anal. Biochem., 89 : 235-246.

[9] Farriol M., Segovia ST., Venero Y and Orta X. (2001). Role of polyamines in cell proliferation in a colon carcinoma cell line. Nutrition, 17 : 934-938.

[10] Leveque J, Foucher F, Bansard J, Havouis R, Grall J. and Moulinoux J. (2000) Polyamine profiles in tumor, normal tissue of the homologous breast, blood, and urine of breast cancer sufferers. J. Breast Cancer. Res. Treat. 2: 99-105.

[11] Masaaki Miyata, Masayuki Furukawa, Koichi Takahashi, Frank J, Gonzalez and Yasushi Yamazoe. (2001) Mechanism of 7, 12 Dimethyl benz (a) anthracene induced immunotoxicity. Role of metabolic activation at the target organ. Jpn. J. Pharmacol. 80: 302309.

[12] Mukherjee K, Roy S, Chanda R and Ganguly AK (1993). Putrescine GABA profile in brain tissue of mice treated with dimethylbenzathracene. Med. Sci. Res., 21; 95-96. 
[13] Rawal UM, Patel US, Rao GN. and Desai RR. (1977). Clinical and biochemical studies on cateractous human lens III. Quantitative study of protein, RNA and DNA. Arogya J. Health Sci., 3 :69-72.

[14] Schipper R, Penning L. and Verhofstad A. (2000). Involvement of polyamines in apoptosis. Facts and controversies : effectors or protectors ? Semin Cancer Biol. 10: 55-68.

[15] Schneider WC. (1957). In: Methods in Enzymology, Colowick S.P. and Kaplan N.O. (eds.), Vol.III, Academic Press, New York, 680 .

[16] Stjernborg L, Heby O, Mamont P and Persson L (1993). Polyamine mediated regulation of Sadenosylmethionine decarboxylase expression in mammalian cells. Studies using 5' ([(2)-4- amino-2-butenyl] methylamino)-5' - deoxyadenosine, a suicide inhibitor of the enzyme. Eur.J.Biochem., 214(3): 671-676.

[17] Sunkara PS, Baylin SB. and Luk GD. (1987). Inhibitors of polyamine biosynthesis : Cellular and invivo effects on tumor proliferation. In: P.P. Mc Cann, A.E. Pegg. and A. Sjoerdsma (eds), Inhibition of polyamine metabolism. Significance and Basis for new therapies. San Diego: Academic Press; 121-140.

[18] Uma Devi P. (1996) Withania somnifera Dunal (Aswangandha): potential plant source of apromising drug for cancer chemotherapy and radiosensitization. Indian journal of Experimental biology, 34 : 927-932. 